\title{
MGMT Gene Promoter Hypermethylation
}

National Cancer Institute

\section{Source}

National Cancer Institute. MGMT Gene Promoter Hypermethylation. NCI Thesaurus.

Code C139588.

A genetic finding indicating an excess of DNA methylation in the promoter region of the MGMT gene. 